\title{
Neck pain: global epidemiology, trends and risk factors
}

\author{
Somaye Kazeminasab ${ }^{1,2}$, Seyed Aria Nejadghaderi ${ }^{3,4}$, Parastoo Amiri ${ }^{1,2}$, Hojjat Pourfathi ${ }^{5}$, \\ Mostafa Araj-Khodaei ${ }^{1,6}$, Mark J. M. Sullman ${ }^{7,8}$, Ali-Asghar Kolahi ${ }^{9^{*}}$ and Saeid Safiri ${ }^{10,11^{*}}$ (D)
}

\begin{abstract}
Background: Neck pain is one of the most common musculoskeletal disorders, having an age-standardised prevalence rate of 27.0 per 1000 population in 2019. This literature review describes the global epidemiology and trends associated with neck pain, before exploring the psychological and biological risk factors associated with the initiation and progression of neck pain.
\end{abstract}

Methods: The PubMed database and Google Scholar search engine were searched up to May 21, 2021. Studies were included that used human subjects and evaluated the effects of biological or psychological factors on the occurrence or progression of neck pain, or reported its epidemiology.

Results: Psychological risk factors, such as long-term stress, lack of social support, anxiety, and depression are important risk factors for neck pain. In terms of the biological risks, neck pain might occur as a consequence of certain diseases, such as neuromusculoskeletal disorders or autoimmune diseases. There is also evidence that demographic characteristics, such as age and sex, can influence the prevalence and development of neck pain, although further research is needed.

Conclusions: The findings of the present study provide a comprehensive and informative overview that should be useful for the prevention, diagnosis, and management of neck pain.

Keywords: Neck pain, Epidemiology, Risk factor, Narrative review

\section{Background}

Neck pain is a multifactorial disease, and is a major problem in modern society. Although neck pain may not be the most common musculoskeletal disorder, it is still very important $[1,2]$. The economic burden of neck pain is remarkable and includes treatment costs, reduced productivity and job-related problems. In 2016, among the 154 conditions, low back and neck pain had the highest health care spending in the United States

\footnotetext{
*Correspondence: a.kolahi@sbmu.ac.ir; safiris@tbzmed.ac.ir

${ }^{9}$ Social Determinants of Health Research Center, Shahid Beheshti University of Medical Sciences, Tehran, Iran

${ }^{10}$ Neurosciences Research Center, Aging Research Institute, Tabriz University of Medical Sciences, Tabriz, Iran

Full list of author information is available at the end of the article
}

with an estimated $\$ 134.5$ billion [3]. In 2012, neck pain was responsible for job absences among 25.5 million Americans, who missed an average of 11.4 days of work [4]. In 2017, the global age-standardised prevalence and incidence rate of neck pain were 3551.1 and 806.6 per 100,000 , respectively [5].

There is no one definitive treatment for neck pain. However, different pharmacological and non-pharmacological treatments have been recommended, including laser therapy, massage, acupuncture, yoga, and aquatic therapy [6-8].

The present study aims to provide a narrative review of the most recent data on the epidemiology and trends associated with neck pain. This review will highlight gaps in our knowledge in order to stimulate and focus future original author(s) and the source, provide a link to the Creative Commons licence, and indicate if changes were made. The images or other third party material in this article are included in the article's Creative Commons licence, unless indicated otherwise in a credit line to the material. If material is not included in the article's Creative Commons licence and your intended use is not permitted by statutory regulation or exceeds the permitted use, you will need to obtain permission directly from the copyright holder. To view a copy of this licence, visit http://creativecommons.org/licenses/by/4.0/. The Creative Commons Public Domain Dedication waiver (http://creativeco mmons.org/publicdomain/zero/1.0/) applies to the data made available in this article, unless otherwise stated in a credit line to the data. 
research, as well as to help healthcare policy makers and clinicians prevent and control this disease.

\section{Methods}

The PubMed database and the Google Scholar search engine were searched up to May 21, 2021 using the following key words: ("neck pain" OR "neck ache" OR "cervical pain" OR "cervicalgia" OR "cervicodynia") AND ("epidemiology" OR "global burden of disease" OR "risk factor" OR "biologic factor" OR "psychologic factors" OR "gender" OR "age" OR "genetic" OR "anxiety" OR "depression" OR "stress" OR "neuromusculoskeletal disorder" OR "autoimmune disease" OR "sleep disorder" OR "behavior" OR "social support"). No search filters, for example publication type or date, were used. Our inclusion criteria were studies that were conducted on human subjects and evaluated the effects of biological (e.g. age, gender, and genetic) or psychological (e.g. psychosocial stress, anxiety, and depression) factors on the occurrence or progression of neck pain. In addition, those articles that reported the epidemiological characteristics or burden of neck pain at the global, regional, or national level were also included. In order to consider and address potential biases, two of the authors independently evaluated and reviewed the studies, based on the inclusion criteria, and any discrepancies were resolved by discussion. The data analysis used a qualitative methodology with a narrative synthesis.

\section{Main text}

\section{Global epidemiology and trends}

In 2017, the East Asia and Andean Latin America regions had the highest and lowest age-standardised incidence rates, with 1029.0 (910.5 to 1166.1 ) and 624.0 (550.3 to $708.3)$ per 100,000 population, respectively. Scandinavian countries, in particular Norway, Finland, and Denmark, had the highest prevalence of neck pain, while Djibouti and South Sudan had the lowest prevalence rates. Over the period 1990-2017, High-income North America had the largest increase in both the age-standardised incidence $(3 \%(-2$ to 7.8$))$ and prevalence rates $(4.1 \%(-2$ to 11.1)), whereas Australasia had the largest decrease in both the incidence $(-1.1 \%(-1.8$ to -0.4$))$ and prevalence rates $(-1.4 \%(-2.1$ to -0.7$))$ over this period (Figs. 1 and 2) [5].

In 2017, the national age standardised point prevalence of neck pain ranged from 2443.9 to 6151.2 cases per 100,000 population. As shown in Fig. 3, the countries with the highest age standardised point prevalence estimates (per 100,000) were Norway (6151.2 (5382.3 to 6959.8)) and Finland (5750.3 (5058.4 to 6518.3)), while the lowest estimates were found in Djibouti (2443.9 (2146.4 to 2771.9)) and South Sudan (2449.8 (2149.8 to 2781.1)).

Also in 2017, the national age standardised annual incidence of neck pain ranged from 599.6 to 1145 cases per 100,000 population. Figure 4 shows that the highest

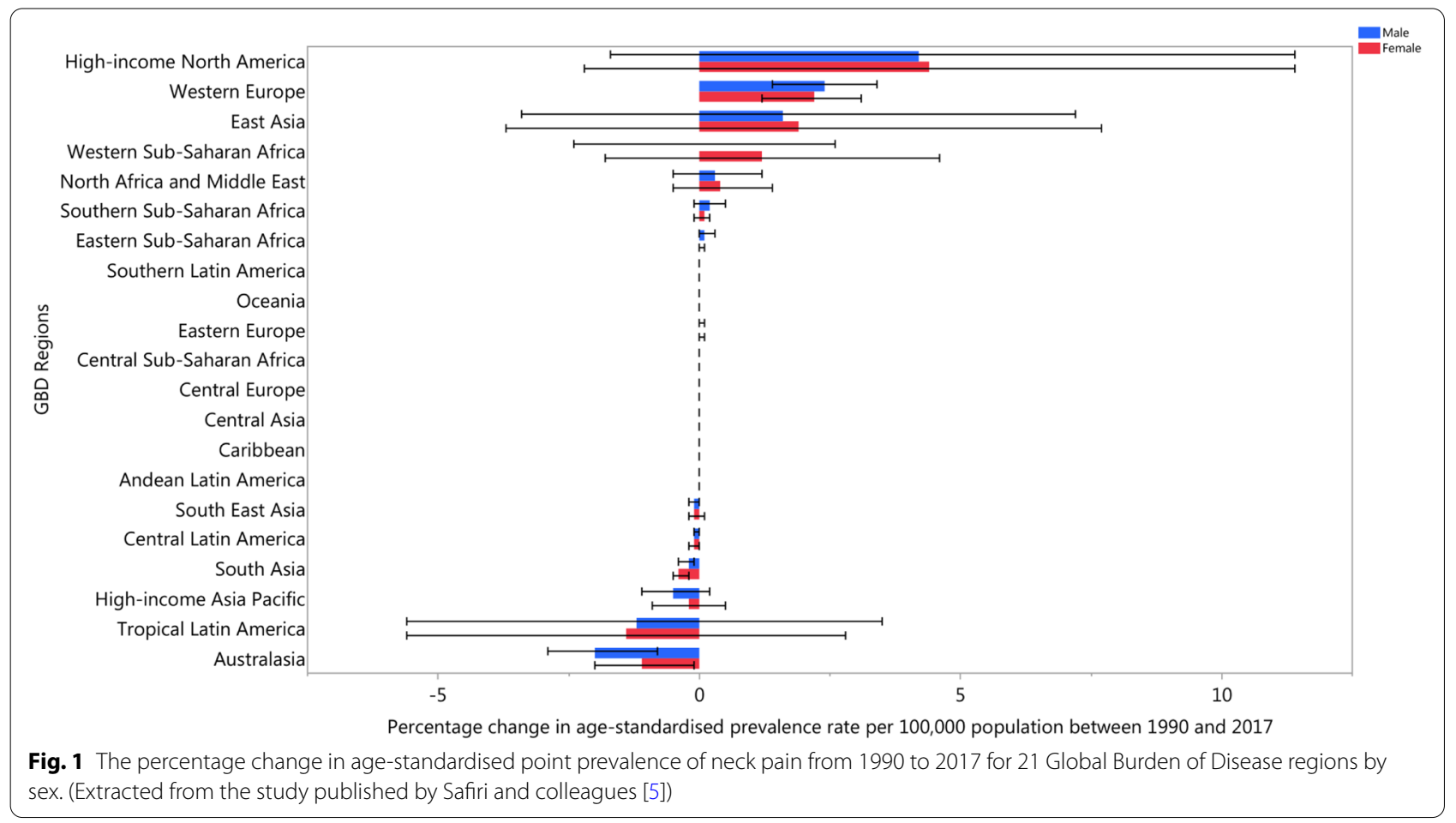



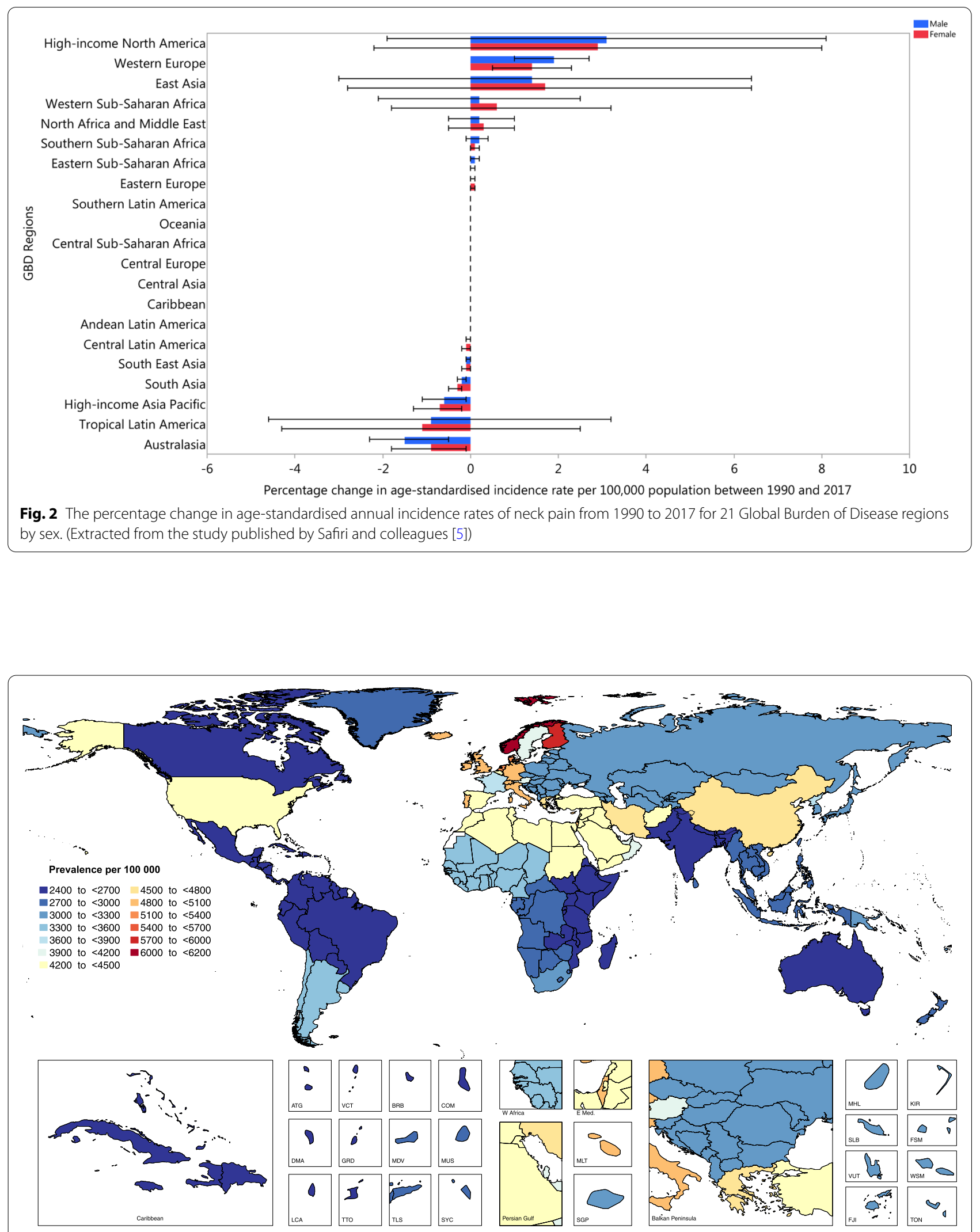

Fig. 3 Age standardised point prevalence of neck pain per 100,000 population in 2017, by country. (Extracted from the study published by Safiri and colleagues [5]) 


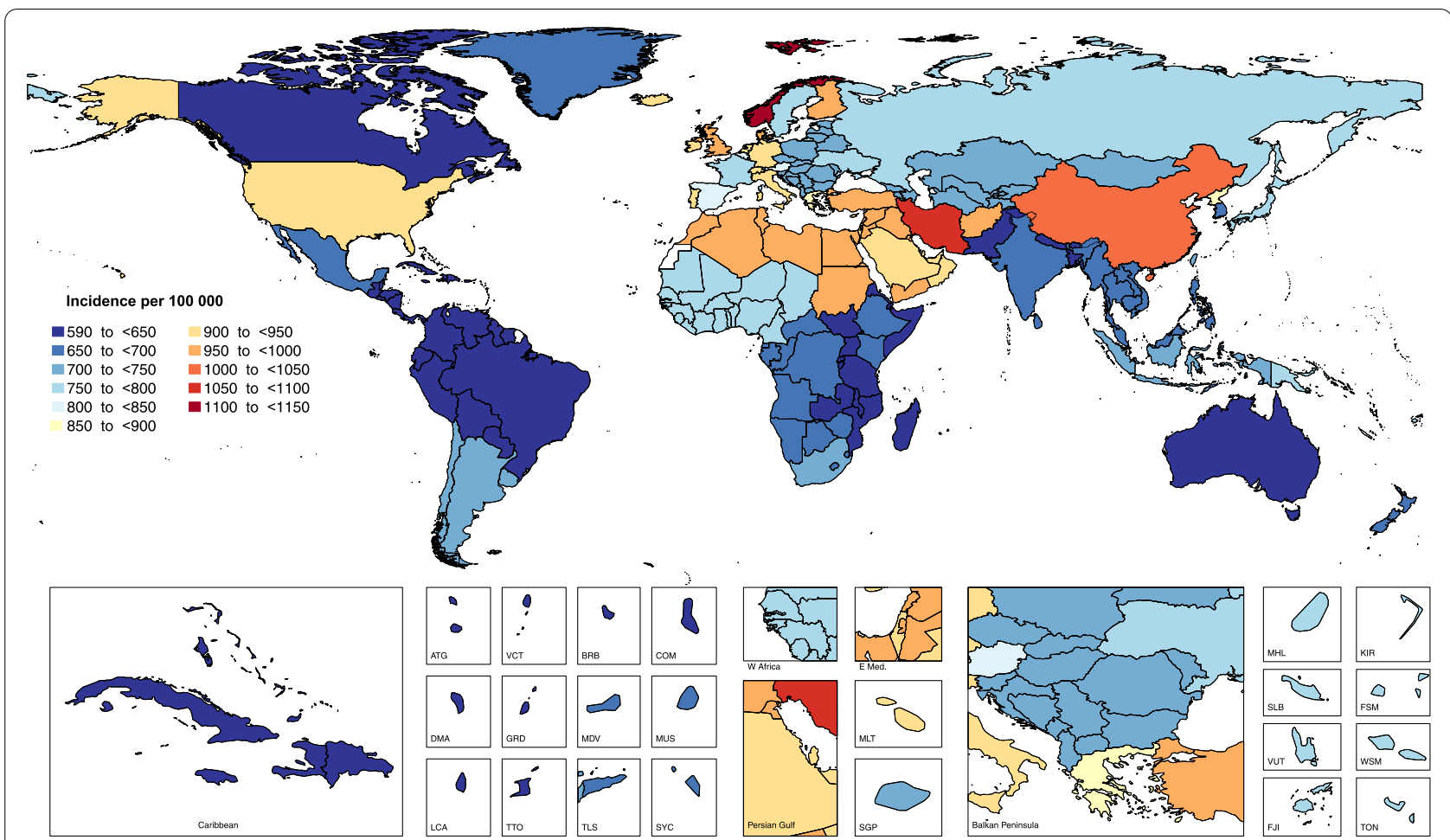

Fig. 4 Age standardised annual incidence of neck pain per 100,000 population in 2017, by country. (Extracted from the study published by Safiri and colleagues [5])

rates were found in Norway (1145 (1008.8 to 1304.9)) and Iran (1055.5 (927.8 to 1199.6)), while the lowest estimates were found in Canada (599.6 (528.8 to 679.1)) and Bhu$\tan (612.4$ (542.1 to 696.3)) (Fig. 4).

The burden of neck pain was higher in females than among males. In 2017, the number of neck pain cases in females was 166.0 million (118.7 to 224.8), while for males it was 122.7 million (87.1 to 167.5) [5]. Furthermore, the number of years lived with disability (YLDs) from neck pain was higher in females (16.4 million (10.0 to 25.1) than among males (12.2 million (7.4 to 18.9) [5]. Globally, in 2017 the age-standardised prevalence of neck pain increased with age up to 70-74, and then decreased with advancing age (Fig. 5). The YLDs pattern across the age groups was relatively similar to that of the estimated point prevalence [5].

\section{Risk factors}

Neck pain is a multifactorial disease. Several populationbased studies have explored the role of various modifiable and non-modifiable risk factors for neck pain, such as advanced age, being female, low social support, and a history of neck or lower back pain [9-12]. Since there is a tendency for neck pain to become a chronic problem, it is important to identify risk factors in order to enable prevention and early diagnosis [12]. As neck pain is a multifactorial disease, there are a number of risk factors which can contribute to its development. There is, however, more evidence for some risk factors, such as lack of physical activity, duration of daily computer use, perceived stress and being female [13]. Identifying protective or risk factors, triggers and outcomes can help guide the prevention, diagnosis, treatment, and management of neck pain. The following section describes the research evidence of a number of psychological and biological factors associated with neck pain.

\section{Psychological factors}

The literature demonstrates a clear link between psychological variables and neck/back pain $[14,15]$. A study based on the China Mental Health Survey showed that the prevalence of chronic back or neck pain among people with any type of mental disorders was more than twice that of those without a mental disorder, with a particularly high prevalence among those with mood disorders [16]. Prospective investigations have demonstrated that psychological variables are related to the onset and severity of pain (i.e. acute, sub-acute, and chronic). Stress, distress, anxiety, mood and emotions, cognitive functioning, and pain-related behaviors have all been found to be important factors in the development of neck pain. Although there is not much evidence for personality 


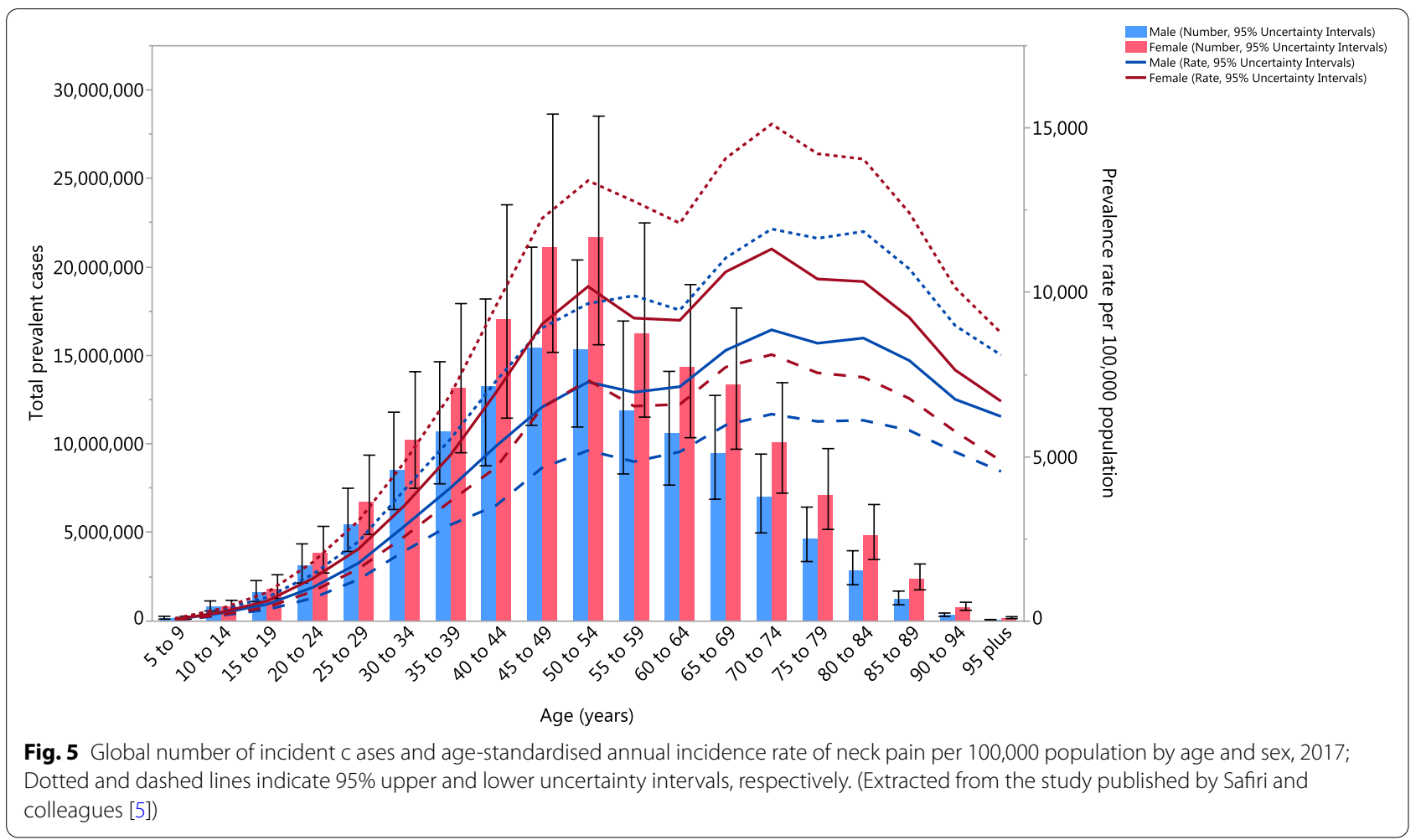

factors like abuse, they can also be potential risk factors $[14,15]$.

Overall, factors such as stress, pain catastrophizing, depressive symptoms, low sleep quality, and alcohol consumption might play some role in changing the central pain processing within the spine, brainstem, or cortical levels, which can manifest as remote hyperalgesia [17]. However, further investigations are needed into the role that these cognitive, affective, and lifestyle factors have in central pain processing in non-traumatic neck pain [17]. The four psychological domains (i.e. cognitions, emotions, social and behavioral domains) involved in neck pain were carefully described and explored in depth. Firstly, there is a cognitive component that is comprised of attitudes, beliefs, and cognitions in relation to pain, disability, and perceived health. A second theme is the emotional dimension, in which distress, anxiety, and depression are the most important variables. Thirdly, there is a social dimension, where family and work issues seem to be related to neck and back pain, although the data are less convincing. Finally, a behavioral domain has also emerged, in which coping, pain behaviors, and activity patterns are important elements [14, 18-21].

\section{Stress}

Stress is related to pain and disability $[14,15]$. Perceived stress is a risk factor for neck pain $[19,22]$. At least two investigations, with fair methodological quality, have found that adolescents with neck pain had significantly more symptoms of stress than adolescents without neck pain, and that permanent and/or regular feelings of stress was significantly associated with an increased odds of reporting neck pain [23]. Stress may contribute to altered central pain processing at the spinal, brainstem, or cortical levels, which may present as remote hyperalgesia - a condition in which individuals experience an enhanced sensitivity to pain [17, 24]. Moreover, stress acts as a mediator between pain and disability $[25,26]$.

\section{Anxiety}

Anxiety is related to different kinds of chronic pain (e.g., neck pain), as well as disability $[14,15,27,28]$. Neck pain has been found to be comorbid with anxiety [28-30]. Trait and state anxiety were investigated using two different measurement instruments and the researchers found that adolescents with neck pain had higher levels of trait and state anxiety than adolescents without neck pain [23]. Furthermore, anxiety disorders were found to be the second most common comorbid disease associated with neck pain, and specific phobias were the most prevalent problem among those with anxiety disorders [16].

An association between lower pressure pain thresholds (PPTs) and increased levels of anxiety have also been reported. PPTs have been found to be associated with 
pain intensity, frequency, duration, and disability due to neck pain [31]. People with neck pain have reported higher levels of anxiety [31], and anxiety has also been found to exacerbate pain and disability [25]. However, there are also some discrepancies in the findings, with one study on the components of psychological distress (e.g., stress, anxiety, and depression) reporting that anxiety was not a mediator for pain and disability [26]. Survey-specific estimates have also revealed some inconsistencies in the comorbidity between spinal pain, both back and neck pain, and anxiety disorders. For instance, only 10 out of 17 surveys showed significantly increased odds ratios for chronic neck pain among those with generalized anxiety disorder and agoraphobia/panic disorder. Therefore, there are some variations in the size of this association between studies and between countries, although chronic spinal pain seems to increase the likelihood of comorbid anxiety disorders.

The research has also shown that some specific types of anxiety disorders are more strongly associated with spinal pain than others. Generalized anxiety disorder and post-traumatic stress disorder (PTSD) are more likely to be comorbid with spinal pain than social phobia or panic disorder/agoraphobia [28, 30, 32]. However, research has found neck pain to be more common in patients with mood disorders than among those with specific anxiety disorders [30].

\section{Depression}

The relationship between depression and neck pain appears to be bidirectional [33]. Mood disorders, especially depression, have been found to be related to chronic neck pain and disabilities [14, 27, 30, 32]. Neck pain is also commonly reported in individuals with underlying depression [13, 29, 34]. Furthermore, a metaanalysis reported that symptoms of depression were associated with high morbidity in neck pain patients [21]. Moreover, a review article suggested that the strongest psychosocial risk factors among respondents with chronic back or neck pain were depressed mood [12] and major depression [30]. A survey study in China showed that mood disorders have a higher comorbidity with neck pain than other mental disorders, and that major depression had the highest comorbidity among all mood disorders [16]. Furthermore, seven studies with fair methodological quality, investigated depression using a total of six different measurement instruments and all studies found that adolescents with neck pain had more depressive symptoms than asymptomatic adolescents [23]. In fact, depressive symptoms may affect central pain processing at the spinal, brainstem or cortical levels, which can be manifested as remote hyperalgesia [17, 35]. Depression and pain might also be risk factors for each other [21]. Several studies have pointed out that psychological stress and the potential obstacles caused by pain may produce immunological changes that eventually results in depression and anxiety [21]. Studies have also found that depression acts as a mediator between pain and disability $[25,26]$.

\section{Cognitive variables}

Cognitive factors (i.e. attitudes, cognitive style, and fearavoidance beliefs) have been linked to increased pain, such as neck pain and disability [14, 15, 27]. Pain cognitions, like catastrophizing and self-perceived poor health, are related to pain and disability [14], as are fear-avoidance beliefs and passive coping [14]. Pain catastrophizing, which is a cognitive factor, may also contribute to altered central pain processing at several levels (i.e., the spinal, brainstem or cortical levels), which may be exhibited as remote hyperalgesia $[17,36]$. Research has found that adolescents with neck pain had higher levels of catastrophizing, compared to adolescents without neck pain [23]. In general, people with neck pain report higher levels of catastrophizing than those without neck pain [31].

Another significant cognitive factor is self-efficacy, which has been shown to be related to neck pain [27]. Low pain self-efficacy has been found to be associated with greater functional disability in patients with neck pain [27], although an article by Andias et al. showed that the difference in self-efficacy levels among adolescents with and without neck pain remains controversial [23].

Low resilience is another psychological factor which has been found to be associated with greater functional disability in patients with neck and back pain [27].

\section{Sleep problems}

The relationship between sleep quality and neck pain is bidirectional, as both can lead to the other [37]. Five investigations, including one longitudinal and four crosssectional studies, most of which were fair methodological quality, assessed sleep using a total of six different measurement instruments and found some evidence that insufficient quantity and quality of sleep were significantly associated with increased odds of having neck pain [23]. Therefore, sleep management might be a promising intervention for decreasing pain sensitivity and increasing pain modulatory capacity $[17,38]$.

There is research to suggest that females with low sleep quality are at a higher risk of neck pain onset, but the findings in male have been inconsistent. Furthermore, one high-quality study found no significant relationship between sleep and shoulder pain, indicating that there is either weak evidence or no higher risk [39-41]. Furthermore, poor sleep quality can lead to an increase in the 
symptoms of depression for people with high intensity neck pain [33].

\section{Social support}

Loneliness is an important factor in early adulthood and its relationship with neck pain should be further investigated [13]. Neck pain has been found to have a positive relationship with poor general social support [27] and poor social support at work [2]. A negative relationship between neck complaints and actively seeking social support has also been reported [42].

\section{Personality}

No evidence exists to support the theory of a "pain prone" personality. Furthermore, findings on the relationship between personality traits or disorders and neck pain have been contradictory [14].

\section{Behavior}

Dangerous behaviors, digital habits, and abnormal eating-related behaviors, such as food insecurity, are common in early adulthood and these behaviors may also contribute to the development of neck pain [13]. Coping styles are the typical manner in which individuals confront and deal with stressful situations, including illness or pain. There are three basic coping styles, which are task-oriented, emotion-oriented, and avoidance-oriented [43]. The relationship between the different coping strategies and pain has been investigated by several studies [44, 45]. Individuals with an avoidance-oriented strategy have a longer duration of neck complaints, since when faced with a problematic situation they seek distraction, avoid thinking about their problem and try to feel better by smoking, drinking, or relaxing [42]. Furthermore, those who react with annoyance or anger tend to have a longer duration of neck complaints [42]. In contrast, there is a negative relationship between seeking social support and neck complaints. In fact, people with neck problems complain of neck pain for a shorter period of time when they share their concerns with others and receive social support and understanding [42].

\section{Work-related factors}

In a review, the most commonly reported risk factor was working in awkward/sustained postures [12]. Work and study time, workload and body position at work are the most important contributors to neck pain [13]. Workplace characteristics, such as perceived job demands, effort-reward imbalance and coworker support were all significant risk factors [12]. Furthermore, research has found neck pain to be related to low job control, routine work, lack of decision making opportunities, low ability to influence working conditions, low job satisfaction, and high job strain [2]. Furthermore, high job demands and low coworker support are independent risk factors for neck pain and there is also evidence that low decisionmaking authority is a risk factor for neck pain [46].

There is some evidence that high skill discretion has a protective effect from neck pain [2]. However, working with a computer is considered to be an occupational condition that causes neck pain [19]. Several factors have been suggested to play key roles in the development of computer-related neck pain, including posture, duration working at a computer, psychological stress, repetitive movements, prolonged static loads, and the psychosocial effects of the working environment [19]. Furthermore, there is also an association between neck pain and eyestrain, regardless of other conditions [19].

\section{Neuromusculoskeletal disorders}

There are a number of diseases and ailments which have been identified as contributing to neck pain. Neuromusculoskeletal disorders affect the bones, muscles, and nerves, and can manifest themselves in several ways. Neck pain is one of the most common and obvious complaints of patients with disorders, such as cervical spondylosis, fibromyalgia, cervical radiculopathy, and whiplash-associated disorders (WADs) [47, 48].

Cervical spondylosis is an umbrella term for a cluster of abnormalities, all of which involve progressive degenerative changes that affect all components of the cervical spine, which is the most frequent neuromusculoskeletal cause of neck pain [49]. In general, neck or occipital pain is known as the first clinical manifestation of degenerative cervical spondylosis. Several factors can lead to cervical spondylosis, such as a congenital narrowing of the cervical spinal canal, cervical osteoarthritis, neck arthritis, trauma, and wearing of the spinal disks. The likelihood of developing cervical spondylosis increases with age, especially after the fourth decade of life, and its progressive nature eventually leads to the involvement of more than one intervertebral disc [50]. The severity of a patient's signs and symptoms are determinative of the treatment strategy. In addition to pain relievers, noninvasive pain relief techniques (e.g., physical therapy and neck mobilisation/manipulation), and medications can help ease neck pain from cervical spondylosis. Surgical intervention is also effective in preventing the progression of neurologic decline [51].

Fibromyalgia is characterized by chronic widespread musculoskeletal pain and additional symptoms, such as extreme tiredness, sleep disturbance, cognitive dysfunction, and mood problems. Previous studies have shown that genetic and epigenetic factors have a critical role in disease susceptibility. While the signs and symptoms vary 
greatly, neck pain is the most frequent complaint among patients [52, 53].

Cervical radiculopathy is a type of neck disorder that results from the compression or irritation of the nerve roots in the cervical spine. Nerve root compression can occur due to spondylosis, instability, trauma, or rarely tumors. The clinical manifestations of radiculopathy are broad, but neck and shoulder pain are the primary complications in patients with cervical radiculopathy. The differential diagnosis of cervical radiculopathy, includes cardiac pain, musculoskeletal diseases, infections, and malignancies [54, 55].

Whiplash-associated disorders (WAD) are characterized by a collection of symptoms affecting the neck and are triggered by sudden acceleration-deceleration movements. WADs are characterized by several clinical complications, including neck pain, nonspecific headache, dizziness, and temporomandibular joint pain that are triggered by a sudden force which stretches the neck muscles and tendons. The prevalence of WADs varies around the world, but up to $50 \%$ of patients develop chronic pain. Therefore, the prevalence of cervical facet pain is high in whiplash patients [56-58].

\section{Autoimmune diseases}

Autoimmune diseases are a chronic and clinically heterogeneous group of diseases that occur in immunocompromised individuals. Autoimmune diseases affect various organs and tissues throughout the body. In some autoimmune diseases, the muscles, joints, and nerves can be the target of the immune system, so they are also likely to affect the cervical spine. The most important autoimmune diseases are rheumatoid arthritis, polymyalgia rheumatic, multiple sclerosis (MS), ankylosing spondylitis, systemic lupus erythematosus (SLE), myositis, and psoriatic spondylitis.

Rheumatoid arthritis is a chronic inflammatory disease that primarily affects the bones, peripheral joints, and ligaments, although it can also affect almost every system. Peripheral joint swelling is a common early symptom, and chronic inflammation of the cervical spine is the second most common feature of rheumatoid arthritis, affecting more than half of all patients with rheumatoid arthritis. Neck pain is one of the earliest symptoms to indicate cervical spine involvement in a patient's rheumatoid arthritis. Nevertheless, occipital headaches and other neurological symptoms may also present in patients with cervical spine involvement $[59,60]$.

Polymyalgia rheumatica is a relatively common chronic inflammatory disorder that is often associated with giant cell arteritis and is characterized by widespread aches and stiffness of the neck, shoulder, and hip area. The average age of patients with polymyalgia rheumatic is 70 and it is rarely found in anyone under 50 years of age. Therefore, it would appear that age-related immune activation, in response to environmental triggers, may contribute to the development of polymyalgia rheumatica $[61,62]$.

MS is considered a multifocal inflammatory autoimmune disease that affects the central nervous system (CNS) and often results in the patient experiencing chronic pain. There are a number of different factors, such as age, sex, disease duration, depression, and fatigue, which cause the prevalence of neck pain to differ widely in patients with MS. Neck pain in MS patients can be due to immobility or Lhermitte's sign, which is defined as a transient short-lasting sensation related to neck movement [63].

Ankylosing spondylitis is a progressive and debilitating form of arthritis that mainly results in inflammation of the joints of the spine. Neck pain is also common in these patients, due to the inflammation of the cervical spine. Recent genetic studies have identified several genes and multiple pathways involved in ankylosing spondylitis. In particular, the activation of specific immune pathways plays a critical role in ankylosing spondylitis pathogenesis [64].

SLE is a severe systemic autoimmune disease that can affect almost any part of the body. Therefore, chronic pain and fatigue are very common in patients with SLE. Inflamed muscles can cause neck and back pain in SLE patients [65].

Myositis is a rare chronic systemic autoimmune disease that is characterized by profound muscle inflammation and progressive muscle weakness. Myositis typically bilaterally affects the skeletal muscles, including the neck, shoulders, hips, and back muscles. Myositis can affect people of any age and, similar to most autoimmune diseases, there is a greater prevalence of myositis in females than among males [66].

Psoriatic arthritis refers to a group of chronic inflammatory joint diseases that develop in some people with psoriasis. Psoriatic spondylitis is a subtype of psoriatic arthritis that affects the spine and causes pain and stiffness in the back and neck [67].

\section{Genetic}

Genetic susceptibility plays an important role in the development of complex diseases. Unraveling the genetic contributions to complex traits is one of the greatest challenges facing modern medical genetics. The identification of the genetic determinants of complex diseases can help provide some understanding of the pathogenesis of the disease. Twin studies and genome-wide association studies (GWASs) can help to develop insights into the genetic architecture of diseases. 
Several population-based twin studies confirm the genetic influence on neck pain. Twin cohort studies compare the concordance rate among monozygotic (MZ) and dizygotic (DZ) twins. The greater similarity of $\mathrm{MZ}$, than DZ, demonstrates the strong genetic contribution to the development of neck pain [68-73]. However, genetic factors seem especially important in the development of neck pain in early adolescence, while their influence could be considered negligible at older ages [68, 72, 74].

There are very few studies which have attempted to unravel the genetic basis of neck pain. GWASs are a powerful approach to identify the genetic loci that predispose individuals to complex diseases. A recent GWAS of the United Kingdom (UK) Biobank participants revealed that there are three genetic loci associated with neck or shoulder pain, including an intergenic region in chromosome 17 for rs12453010, FOXP2 gene in chromosome 7 for rs34291892 and rs62053992 in LINC01572 gene in chromosome 16 [75]. However, to the best of our knowledge, only one GWAS study has been published to date and further evidence should be sought to confirm the genetic correlations between these loci and neck pain.

\section{Gender}

The results of several systematic reviews have demonstrated that gender is a well-studied but ambiguous risk factor for neck pain. Previous studies have considered being female to be a significant risk factor for developing neck pain $[11,76]$. Nevertheless, in contrast to previous neck pain articles, recent epidemiological studies have found no meaningful sex differences in the prevalence, incidence, and years lived with disability across the age groups in patients with neck pain $[12,13,77]$. However, the point prevalence of neck pain was higher in females across all age groups [5]. These contradictions are also evident from the articles that report being female to be a weak predictor of developing neck pain, since the age of onset is also important and this may differ between males and females. For this reason, sex-specific meta-analyses are needed to clarify the ambiguous association between sex and neck pain.

\section{Age}

Aging is the most important risk factor for most chronic pain, so identifying protective and risk factors is critical for raising awareness about effective preventative measures and educational interventions for high-risk groups [11]. The normal anatomy of the cervical spine changes at advanced ages, which can cause neck pain and long-term disability. Neck pain is common among adults, although it can occur at any age. According to the Global Burden of Diseases 2017 study, the point prevalence of neck pain peaked during the middle ages and declined thereafter, with the highest burdens being in the 45-49 and 50-54 age groups for men and women, respectively [5].

\section{Discussion}

In this narrative review, we aimed to provide the most recent data on the epidemiology and trends associated with neck pain, and its attributable risk factors, in order to highlight gaps in our knowledge and to stimulate and focus further research, as well as to help healthcare policy makers and clinicians preventing and controlling this disease. Our narrative review showed that the global epidemiology of neck pain has not changed substantially over the last 30 years. We also found that both biological and psychological risk factors, like age, genetics, past medical history of musculoskeletal disorders, stress, anxiety, and depression can promote the occurrence of neck pain. However, the effects of some other factors, like personality disorders and gender need to be investigated more deeply.

Efforts to identify the potential risk factors for neck pain have been underway for decades. The effects of different types of psychological and biological factors have been presented previously [14], but these often had small sample sizes $[9,76]$. Furthermore, there is a paucity of primary research conducted in several areas of the world, which could lead to limitations in the data sources used to estimate the burden of neck pain in different regions [78]. This article placed the risk factors into two main categories, which were psychological and biological factors. However, there are other methods of categorizing the risk factors, such as according to the level of association and preventative potential. For example, risk factors could be divided into those that have a high correlation and are difficult or impossible to prevent, risk factors with a strong correlation that are preventable, and factors that have a low correlation with neck pain. Furthermore, as previously mentioned, several factors (e.g., sleep disturbances and depression) have a bidirectional association with neck pain.

The risk factors for neck pain can be placed into three categories, which are physical, psychosocial, and individual-related risk factors. In a review by Kim et al., they found that most risk factors for neck pain were related to psychosocial, rather than physical characteristics [12]. Neck pain has also been associated with many other comorbidities, such as headaches, dizziness, anxiety, and depression [29]. Nevertheless, there are also some inconsistencies in the research investigating the relationship neck pain has with psychological factors.

There are also several risk factors, like depression and occupational factors that not only have a strong association with neck pain, but are also preventable. Patients with musculoskeletal disorders are the most common 


\section{Neck pain risk factors}

\section{Psychological}

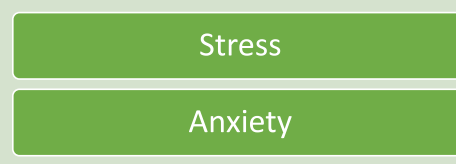

Cognitive variables

Sleep problems

Social support

Personality

Behavior

\section{Biological}

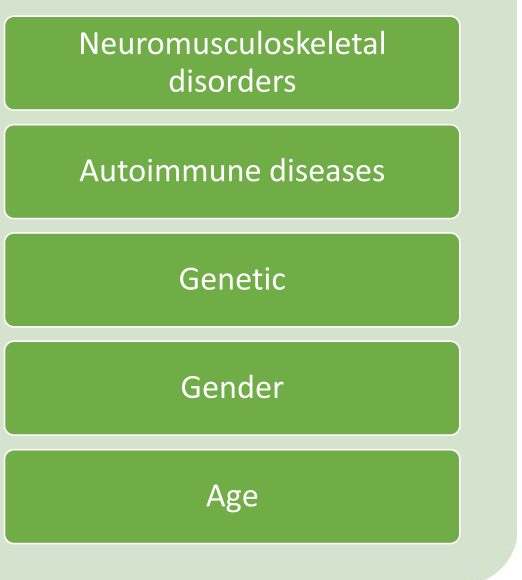

Fig. 6 Summary diagram representing the biological and psychological risk factors of neck pain disorder that might benefit from rehabilitation services (1.71 billion people (95\% UI: 1.68-1.80)) [79]. Furthermore, neck pain is the second most common musculoskeletal disorders with a higher prevalence and incidence among females [5, 79]. Moreover, in 2016, low-back and neck pain were the third largest cause of disabilityadjusted life-years (DALYs) in both sexes (87 million (61 to 114)) [80]. Therefore, finding a cost-effective diagnostic method and treatment strategy is important [81]. Furthermore, improvements in the definition and registration of pain conditions, such as neck pain, could make a large contribution to improving health by providing reliable estimates of the burden of neck pain which can lead to appropriate resource allocation and the implementation of preventative and treatment programs [78].

As previously mentioned, work-related factors (e.g., work load, working on a computer, and study time) are risk factors for the development of neck pain. Nevertheless, children and adolescents are also at a risk of developing neck pain. A cross-sectional study of 207 children and adolescents with non-specific neck pain showed that strong flexion of the neck during studying, sitting, watching television, and using smartphones (or other handheld devices) was associated with neck pain [82]. Furthermore, an article by Scarabottolo et al. revealed that physical inactivity in adolescents was associated with an increase in the risk of cervical pain (odds ratio $(\mathrm{OR})=1.49(1.06-$ 2.10)) [83]. Moreover, using a mobile phone for more than $10 \mathrm{~h}$ per week significantly increased the odds of developing neck pain in students aged 10-17years old $(\mathrm{OR}=2.48$; 95\% CI: 1.29-4.75) [84].

There is now evidence from large epidemiological studies that there is an association between mental disorders and chronic pain. The comorbidity between psychological disorders and pain has implications for the outcome of pain and possibly also for the outcome of the psychological disorders. The relationship between psychological disorders and chronic pain, in the case of co-occurrence, is often complex and cannot be easily interpreted and managed. Although a relationship between chronic pain and psychiatric disorders has been identified, the relationship pain severity has with mood or anxiety disorders is less clear [32]. Furthermore, research in diverse cultures and socioeconomic situations has shown that people with chronic neck pain were more likely to suffer from psychological disorders (e.g., mood, anxiety, and alcohol disorders), compared to those without neck pain [30].

The most prominent limitation of this study is that it had a narrative approach, rather than a systematic review and meta-analysis, so it is difficult to draw precise conclusions. In addition, we only investigated 
biological and psychological risk factors, although there are other potential risk factors, such as a history of previous neck injury, number of children, past medical history of low back pain, and poor self-assessed health [30, 85]. In addition, we acknowledge that some of the topics covered under biological risk factors may not have been completely balanced. Despite these limitations, this comprehensive review has highlighted important gaps in our knowledge and areas that require further research. Future research should be conducted as systematic reviews, with or without meta-analysis, on specific biological (e.g. gender) and psychological (e.g. type of personality) risk factors, especially those with inconsistent findings, in order to clarify whether or not there is an association.

\section{Conclusions}

Neck pain has a high prevalence around the world, although its burden has not changed substantially over the period 1990-2019. Recent literature has shown that psychological factors (e.g., stress, some cognitive factors, and sleep problems) and individual/biological factors (e.g., preexisting neuromuscular or autoimmune disorders, aging, and genetic) both contribute to the development of neck pain (Fig. 6). The relationship between personality types and gender on the risk of neck pain is not yet clear, so further research is needed to investigate the association that neck pain has with gender, personality, and several other psychological factors.

\section{Abbreviations \\ UI: Uncertainty interval; YLD: Years lived with disability; DALY: Disability- adjusted life years; WAD: Whiplash-associated disorder; MS: Multiple sclerosis; SLE: Systemic lupus erythematosus; CNS: Central nervous system; GWAS: Genome-wide association studies; MZ: Monozygotic; DZ: Dizygotic; UK: United Kingdom; PPT: Pressure pain threshold; PTSD: Post-traumatic stress disorder; OR: Odds ratio.}

\section{Acknowledgments}

The authors acknowledge the support received from the Social Determinants of Health Research Center at the Shahid Beheshti University of Medical Sciences.

\section{Author s' cont ributions}

MJMS, AAK and SS conceptualized and designed the study. SK, PA, SAN, HP, and M AK drafted the initial manuscript. All authors reviewed the drafted manuscript for critical content. All authors approved the final version of the manuscript.

\section{Funding}

The present study was supported by the Social Determinants of Health Research Center at the Shahid Beheshti University of Medical Sciences, Tehran, Iran (Grant No. 24280)

\section{Availability of data and materials}

Not applicable.

\section{Declarations}

Ethics approval and consent to participate

Not applicable.

\section{Consent for publication}

Not applicable.

\section{Competing interests}

The authors declare that they have no competing interests.

\section{Author details}

${ }^{1}$ Research Center for Integrative Medicine in Aging, Aging Research Institute, Tabriz University of Medical Sciences, Tabriz, Iran. ${ }^{2}$ Research Deputy, Faculty of Medicine, Tabriz University of Medical Sciences, Tabriz, Iran. ${ }^{3}$ School of Medicine, Shahid Beheshti University of Medical Sciences, Tehran, Iran. ${ }^{4}$ Systematic Review and Meta-analysis Expert Group (SRMEG), Universal Scientific Education and Research Network (USERN), Tehran, Iran. ${ }^{5}$ Department of Anesthesiology and Pain Management, Faculty of Medicine, Tabriz University of Medical Sciences, Tabriz, Iran. ${ }^{6}$ Department of Persian Medicine, School of Traditional Medicine, Tabriz University of Medical Sciences, Tabriz, Iran. ${ }^{7}$ Department of Social Sciences, University of Nicosia, Nicosia, Cyprus. ${ }^{8}$ Department of Life and Health Sciences, University of Nicosia, Nicosia, Cyprus. ${ }^{9}$ Social Determinants of Health Research Center, Shahid Beheshti University of Medical Sciences, Tehran, Iran. ${ }^{10}$ Neurosciences Research Center, Aging Research Institute, Tabriz University of Medical Sciences, Tabriz, Iran. ${ }^{11}$ Social Determinants of Health Research Center, Department of Community Medicine, Faculty of Medicine, Tabriz University of Medical Sciences, Tabriz, Iran.

Received: 20 July 2021 Accepted: 12 December 2021

Published online: 03 January 2022

\section{References}

1. Cohen SP. Epidemiology, diagnosis, and treatment of neck pain. Mayo Clin Proc. 2015;90(2):284-99.

2. Ariëns $G A$, van Mechelen W, Bongers PM, Bouter LM, van der Wal G. Psychosocial risk factors for neck pain: a systematic review. Am J Ind Med. 2001;39(2):180-93.

3. Dieleman JL, Cao J, Chapin A, Chen C, Li Z, Liu A, et al. US health care spending by payer and health condition, 1996-2016. JAMA. 2020;323(9):863-84.

4. Lezin N, Watkins-Castillo S. The impact of musculoskeletal disorders on Americans-opportunities for action. Burden Musculoskelet Dis US Prev Soc Econ Cost. 2016;3.

5. Safiri S, Kolahi A-A, Hoy D, Buchbinder R, Mansournia MA, Bettampadi D, et al. Global, regional, and national burden of neck pain in the general population, 1990-2017: systematic analysis of the Global Burden of Disease Study 2017. BMJ. 2020;368.

6. Skelly AC, Chou R, Dettori JR, Turner JA, Friedly JL, Rundell SD, et al. AHRQ Comparative Effectiveness Reviews. In: Noninvasive nonpharmacological treatment for chronic pain: a systematic review update. Rockville: Agency for Healthcare Research and Quality (US); 2020.

7. Li Y, Li S, Jiang J, Yuan S. Effects of yoga on patients with chronic nonspecific neck pain: a PRISMA systematic review and meta-analysis. Medicine (Baltimore). 2019;98(8):-e14649.

8. Corvillo I, Armijo F, Álvarez-Badillo A, Armijo O, Varela E, Maraver F. Efficacy of aquatic therapy for neck pain: a systematic review. Int J Biometeorol. 2020;64(6):915-25.

9. Genebra CVDS, Maciel NM, Bento TPF, Simeão SFAP, Vitta AD. Prevalence and factors associated with neck pain: a population-based study. Braz J Phys Ther. 2017;21(4):274-80.

10. Hogg-Johnson S, van der Velde G, Carroll LJ, Holm LW, Cassidy JD, Guzman J, et al. The burden and determinants of neck pain in the general population. Eur Spine J. 2008;17(1):39-51.

11. McLean SM, May S, Klaber-Moffett J, Sharp DM, Gardiner E. Risk factors for the onset of non-specific neck pain: a systematic review. J Epidemiol Community Health. 2010;64(7):565-72. 
12. Kim R, Wiest C, Clark K, Cook C, Horn M. Identifying risk factors for first-episode neck pain: a systematic review. Musculoskelet Sci Pract. 2018:33:77-83.

13. Jahre H, Grotle M, Smedbråten K, Dunn KM, Øiestad BE. Risk factors for non-specific neck pain in young adults. A systematic review. BMC Musculoskelet Disord. 2020;21(1):1-12.

14. Linton SJ. A review of psychological risk factors in back and neck pain. Spine. 2000;25(9):1148-56.

15. Martinez-Calderon J, Flores-Cortes M, Morales-Asencio JM, LuqueSuarez A. Which psychological factors are involved in the onset and/or persistence of musculoskeletal pain? An umbrella review of systematic reviews and Meta-analyses of prospective cohort studies. Clin J Pain. 2020;36(8):626-37.

16. Xu Y, Wang Y, Chen J, He Y, Zeng Q, Huang Y, et al. The comorbidity of mental and physical disorders with self-reported chronic back or neck pain: results from the China mental health survey. J Affect Disord. 2020;260:334-41.

17. Xie Y, Jun D, Thomas L, Coombes B, Johnston V. Comparing central pain processing in individuals with non-traumatic neck pain and healthy individuals: a systematic review and meta-analysis. J Pain 2020;21(11-12):1101-24.

18. Ortego G, Villafañe JH, Doménech-García V, Berjano P, Bertozzi L, Herrero $\mathrm{P}$. Is there a relationship between psychological stress or anxiety and chronic nonspecific neck-arm pain in adults? A systematic review and meta-analysis. J Psychosom Res. 2016;90:70-81.

19. Mork R, Falkenberg HK, Fostervold KI, Thorud H-MS. Discomfort glare and psychological stress during computer work: subjective responses and associations between neck pain and trapezius muscle blood flow. Int Arch Occup Environ Health. 2020;93(1):29-42.

20. Baur H, Grebner S, Blasimann A, Hirschmüller A, Kubosch EJ, Elfering A. Work-family conflict and neck and back pain in surgical nurses. Int J Occup Saf Ergon. 2018;24(1):35-40.

21. Liu F, Fang T, Zhou F, Zhao M, Chen M, You J, et al. Association of depression/anxiety symptoms with neck pain: a systematic review and meta-analysis of literature in China. Pain Res Manag. 2018;2018:3259431

22. Grimby-Ekman A, Andersson EM, Hagberg M. Analyzing musculoskeletal neck pain, measured as present pain and periods of pain, with three different regression models: a cohort study. BMC Musculoskelet Disord. 2009:10(1):73.

23. Andias R, Silva AG. Psychosocial variables and sleep associated with neck pain in adolescents: a systematic review. Phys Occup Ther Pediatr. 2020:40(2):168-91.

24. Jennings EM, Okine BN, Roche M, Finn DP. Stress-induced hyperalgesia. Prog Neurobiol. 2014;121:1-18.

25. Lee H, Hübscher M, Moseley GL, Kamper SJ, Traeger AC, Mansell G, et al. How does pain lead to disability? A systematic review and metaanalysis of mediation studies in people with back and neck pain. Pain. 2015;156(6):988-97.

26. Hall AM, Kamper SJ, Maher CG, Latimer J, Ferreira ML, Nicholas MK. Symptoms of depression and stress mediate the effect of pain on disability. Pain. 2011;152(5):1044-51.

27. Ahmed SA, Shantharam G, Eltorai AE, Hartnett DA, Goodman A, Daniels $\mathrm{AH}$. The effect of psychosocial measures of resilience and self-efficacy in patients with neck and lower back pain. Spine J. 2019;19(2):232-7.

28. Gureje O. Comorbidity of pain and anxiety disorders. Curr Psychiatry Rep. 2008;10(4):318-22.

29. Bobos P, MacDermid J, Nazari G, Furtado R. Psychometric properties of the global rating of change scales in patients with neck disorders: a systematic review with meta-analysis and meta-regression. BMJ Open. 2019;9(11):e033909.

30. Demyttenaere K, Bruffaerts R, Lee S, Posada-Villa J, Kovess V, Angermeyer MC, et al. Mental disorders among persons with chronic back or neck pain: results from the world mental health surveys. Pain. 2007;129(3):332-42.

31. Sá S, Silva AG. Repositioning error, pressure pain threshold, catastrophizing and anxiety in adolescents with chronic idiopathic neck pain. Musculoskelet Sci Pract. 2017:30:18-24

32. Kayhan F, Albayrak Gezer I, Kayhan A, Kitiş S, Gölen M. Mood and anxiety disorders in patients with chronic low back and neck pain caused by disc herniation. Int J Psychiatry Clin Pract. 2016;20(1):19-23.
33. Juan W, Rui L, Wei-Wen Z. Chronic neck pain and depression: the mediating role of sleep quality and exercise. Psychol Health Med. 2020:25(8):1029-35.

34. Prins Y, Crous L, Louw Q. A systematic review of posture and psychosocial factors as contributors to upper quadrant musculoskeletal pain in children and adolescents. Physiother Theory Pract. 2008;24(4):221-42.

35. Garrigós-Pedrón M, La Touche R, Navarro-Desentre P, Gracia-Naya M, Segura-Ortí E. Widespread mechanical pain hypersensitivity in patients with chronic migraine and temporomandibular disorders: relationship and correlation between psychological and sensorimotor variables. Acta Odontol Scand. 2019;77(3):224-31.

36. Mason KJ, O'Neill TW, Lunt M, Jones AK, McBeth J. Psychosocial factors partially mediate the relationship between mechanical hyperalgesia and self-reported pain. Scand J Pain. 2018;18(1):59-69.

37. Peterson G, Pihlström N. Factors associated with neck and shoulder pain: a cross-sectional study among 16,000 adults in five county councils in Sweden. BMC Musculoskelet Disord. 2021;22(1):872.

38. Nijs J, Loggia ML, Polli A, Moens M, Huysmans E, Goudman L, et al. Sleep disturbances and severe stress as glial activators: key targets for treating central sensitization in chronic pain patients? Expert Opin Ther Targets. 2017;21(8):817-26.

39. Andreucci M, Campbell P, Dunn KM. Are sleep problems a risk factor for the onset of musculoskeletal pain in children and adolescents? A systematic review. Sleep. 2017:40(7):1-11.

40. Auvinen JP, Tammelin TH, Taimela SP, Zitting PJ, Järvelin M-R, Taanila AM, et al. Is insufficient quantity and quality of sleep a risk factor for neck, shoulder and low back pain? A longitudinal study among adolescents. Eur Spine J. 2010;19(4):641-9.

41. Ståhl M, Kautiainen H, El-Metwally A, Häkkinen A, Ylinen J, Salminen $J$, et al. Non-specific neck pain in schoolchildren: prognosis and risk factors for occurrence and persistence. A 4-year follow-up study. PAIN ${ }^{\circledR}$. 2008;137(2):316-22

42. Buitenhuis J, Spanjer J, Fidler V. Recovery from acute whiplash: the role of coping styles. Spine. 2003;28(9):896-901.

43. Esteve R, Ramírez-Maestre C, López-Martínez AE. Adjustment to chronic pain: the role of pain acceptance, coping strategies, and pain-related cognitions. Ann Behav Med. 2007;33(2):179-88.

44. Wachholtz AB, Pearce MJ, Koenig H. Exploring the relationship between spirituality, coping, and pain. J Behav Med. 2007;30(4):311-8.

45. Murugan S, Saravanan P, Avaiya D, Bawa I, Shah C, Vaghasiya E. Prevalence and Risk Factors for Musculoskeletal Pain and Coping Strategies in School Teachers.J Ecophysiol Occup Health. 2021:2021:6.

46. Ariëns GA, Bongers PM, Hoogendoorn WE, Houtman IL, van der Wal G, van Mechelen W. High quantitative job demands and low coworker support as risk factors for neck pain: results of a prospective cohort study. Spine. 2001;26(17):1896-901.

47. Haldeman S, Carroll L, Cassidy JD. Findings from the bone and joint decade 2000 to 2010 task force on neck pain and its associated disorders. J Occup Environ Med. 2010:52(4):424-7.

48. Moradi-Lakeh M, Forouzanfar MH, Vollset SE, El Bcheraoui C, Daoud F, Afshin A, et al. Burden of musculoskeletal disorders in the eastern Mediterranean region, 1990-2013: findings from the global burden of disease study 2013. Ann Rheum Dis. 2017:76(8):1365-73.

49. Kuo DT, Tadi P. Cervical Spondylosis. [Updated 2021 Sep 29]. In: StatPearls [Internet]. Treasure Island (FL): StatPearls Publishing; 2021. Available from: https://www.ncbi.nlm.nih.gov/books/NBK551557/.

50. Jiang S-D, Jiang L-S, Dai L-Y. Degenerative cervical spondylolisthesis: a systematic review. Int Orthop. 2011;35(6):869-75.

51. Lebl DR, Bono CM. Update on the diagnosis and management of cervical spondylotic myelopathy. J Am Acad Orthop Surg. 2015;23(11):648-60.

52. Björkegren K, Wallander M-A, Johansson S, Svärdsudd K. General symptom reporting in female fibromyalgia patients and referents: a population-based case-referent study. BMC Public Health. 2009;9(1):402.

53. D'Agnelli S, Arendt-Nielsen L, Gerra MC, Zatorri K, Boggiani L, Baciarello $M$, et al. Fibromyalgia: genetics and epigenetics insights may provide the basis for the development of diagnostic biomarkers. Mol Pain. 2019;15:1744806918819944

54. Corey DL, Comeau D. Cervical radiculopathy. Med Clin. 2014;98(4):791-9.

55. Woods BI, Hilibrand AS. Cervical radiculopathy. J Spinal Disord Tech. 2015;28(5):E251-9. 
56. McClune T, Burton AK, Waddell G. Whiplash associated disorders: a review of the literature to guide patient information and advice. Emerg Med J. 2002;19(6):499-506.

57. Pastakia K, Kumar S. Acute whiplash associated disorders (WAD). Open Access Emerg Med. 2011;3:29.

58. Poorbaugh K, Brismée JM, Phelps V, Sizer PS Jr. Late whiplash syndrome: a clinical science approach to evidence-based diagnosis and management. Pain Pract. 2008;8(1):65-89.

59. Gillick JL, Wainwright J, Das K. Rheumatoid arthritis and the cervical spine: a review on the role of surgery, Int J Rheumatol. 2015;2015:252456.

60. Mańczak M, Gasik R. Cervical spine instability in the course of rheumatoid arthritis-imaging methods. Reumatologia. 2017;55(4):201.

61. González-Gay MA, Matteson EL, Castañeda S. Polymyalgia rheumatica. Lancet. 2017;390(10103):1700-12.

62. Guggino G, Ferrante A, Macaluso F, Triolo G, Ciccia F. Pathogenesis of polymyalgia rheumatica. Reumatismo. 2018;70(1):10-7.

63. O'Connor AB, Schwid SR, Herrmann DN, Markman JD, Dworkin RH. Late whiplash syndrome: a clinical science approach to evidence-based diagnosis and management. PAIN ${ }^{\circledR}$. 2008;137(1):96-111.

64. Ranganathan V, Gracey E, Brown MA, Inman RD, Haroon N. Pathogenesis of ankylosing spondylitis — recent advances and future directions. Nat Rev Rheumatol. 2017;13(6):359.

65. Bliddal H, Danneskiold-Samsøe B. Chronic widespread pain in the spectrum of rheumatological diseases. Best Pract Res Clin Rheumatol. 2007:21(3):391-402.

66. McGrath ER, Doughty CT, Amato AA. Autoimmune myopathies: updates on evaluation and treatment. Neurotherapeutics. 2018;15(4):976-94.

67. Cantini F, Niccoli L, Nannini C, Kaloudi O, Bertoni M, Cassara E. Psoriatic arthritis: a systematic review. Int J Rheum Dis. 2010;13(4):300-17.

68. Fejer R, Hartvigsen J, Kyvik KO. Heritability of neck pain: a populationbased study of 33794 Danish twins. Rheumatology. 2006:45(5):589-94.

69. Hartvigsen J, Nielsen J, Kyvik KO, Fejer R, Vach W, lachine I, et al. Heritability of spinal pain and consequences of spinal pain: a comprehensive genetic epidemiologic analysis using a population-based sample of 15,328 twins ages 20-71 years. Arthritis Care Res. 2009;61(10):1343-51.

70. MacGregor AJ, Andrew T, Sambrook PN, Spector TD. Structural, psychological, and genetic influences on low back and neck pain: a study of adult female twins. Arthritis Care Res. 2004;51(2):160-7.

71. Nyman T, Mulder M, Iliadou A, Svartengren M, Wiktorin C. High heritability for concurrent low back and neck-shoulder pain: a study of twins. Spine. 2011;36(22):E1469-76.

72. Ståhl MK, El-Metwally AA, Mikkelsson MK, Salminen JJ, Pulkkinen LR, Rose RJ, et al. Genetic and environmental influences on non-specific neck pain in early adolescence: a classical twin study. Eur J Pain. 2013;17(6):791-8.

73. Wang G, Cao Y, Wu T, Duan C, Wu J, Hu J, et al. Genetic factors of cervical spondylotic myelopathy-a systemic review. J Clin Neurosci. 2017:44:89-94.

74. Hartvigsen J, Pedersen HC, Frederiksen H, Christensen K. Small effect of genetic factors on neck pain in old age: a study of 2,108 Danish twins 70 years of age and older. Spine. 2005;30(2):206-8

75. Meng W, Chan BW, Harris C, Freidin MB, Hebert HL, Adams MJ, et al. A genome-wide association study finds genetic variants associated with neck or shoulder pain in UK biobank. Hum Mol Genet. 2020;29(8):1396-404.

76. Côté P, Cassidy JD, Carroll LJ, Kristman V. The annual incidence and course of neck pain in the general population: a population-based cohort study. Pain. 2004;112(3):267-73.

77. Jun D, Zoe M, Johnston V, O'Leary S. Physical risk factors for developing non-specific neck pain in office workers: a systematic review and metaanalysis. Int Arch Occup Environ Health. 2017;90(5):373-410.

78. Blyth FM, Huckel Schneider C. Global burden of pain and global pain policy_creating a purposeful body of evidence. PAIN. 2018;159(11):S43-8.

79. Cieza A, Causey K, Kamenov K, Hanson SW, Chatterji S, Vos T. Global estimates of the need for rehabilitation based on the global burden of disease study 2019: a systematic analysis for the global burden of disease study 2019. Lancet. 2020:396(10267):2006-17.

80. DALYS GBD, Collaborators H. Global, regional, and national disabilityadjusted life-years (DALYs) for 333 diseases and injuries and healthy life expectancy (HALE) for 195 countries and territories, 1990-2016: a systematic analysis for the global burden of disease study 2016. Lancet. 2017:390(10100):1260-344.
81. Miyamoto GC, Lin CC, Cabral CMN, van Dongen JM, van Tulder MW. Cost-effectiveness of exercise therapy in the treatment of non-specific neck pain and low back pain: a systematic review with meta-analysis. Br J Sports Med. 2019;53(3):172-81.

82. Fares J, Fares MY, Fares Y. Musculoskeletal neck pain in children and adolescents: risk factors and complications. Surg Neurol Int. 2017:8:72.

83. Scarabottolo CC, Pinto RZ, Oliveira CB, Zanuto EF, Cardoso JR, Christofaro DGD. Back and neck pain prevalence and their association with physical inactivity domains in adolescents. Eur Spine J. 2017;26(9):2274-80.

84. Minghelli B. Musculoskeletal spine pain in adolescents: epidemiology of non-specific neck and low back pain and risk factors. J Orthop Sci. 2020;25(5):776-80.

85. Croft PR, Lewis M, Papageorgiou AC, Thomas E, Jayson MIV, Macfarlane GJ, et al. Risk factors for neck pain: a longitudinal study in the general population. Pain. 2001;93(3):317-25.

\section{Publisher's Note}

Springer Nature remains neutral with regard to jurisdictional claims in published maps and institutional affiliations.

Ready to submit your research? Choose BMC and benefit from:

- fast, convenient online submission

- thorough peer review by experienced researchers in your field

- rapid publication on acceptance

- support for research data, including large and complex data types

- gold Open Access which fosters wider collaboration and increased citations

- maximum visibility for your research: over $100 \mathrm{M}$ website views per year

At BMC, research is always in progress.

Learn more biomedcentral.com/submissions 